Thomas Hausmanninger

\title{
Review: Wer hat unseren Kindern das Töten beigebracht? Ein Aufruf gegen Gewalt in Fernsehen, Film und Computerspielen.
}

\begin{abstract}
:
Review of Lt. Col. Dave Grossman and Gloria DeGaetano: Stop Teaching Our Kids to Kill. A Call to Action Against TV, Movie \& Video Game Violence, New York: Crown Publications, 1999, 196 S.

Lt. Col. Dave Grossman, Gloria DeGaetano: Wer hat unserem Kindern das Töten beigebracht? Ein Aufruf gegen Gewalt in Fernsehen, Film und Computerspielen. Mit Beiträgen von Prof. Werner Glogauer, Barbara Supp und Dr. Bruno Sandkühler, Stuttgart: Verlag freies Geistesleben \& Urachhaus 2002, 194 S., € 14,50
\end{abstract}

\section{Author:}

Prof. Dr. Thomas Hausmanninger:

- University of Augsburg, Universitätsstraße 10, 86135 Augsburg, Germany

- Telephone, email and personal homepage: $\mathbf{m}$ + 498215985820 ,

$\triangle$ thomas.hausmanninger@kthf.uni-augsburg.de,

믈 http://www.kthf.uni-augsburg.de/lehrstuehle/sozethik.shtml 
Dave Grossmans und Gloria DeGaetanos schmales Buch teilt mit dem ähnlich schlanken Erfolgsschlager Neil Postmans - „Wir amüsieren uns zu Tode“ - drei zentrale Charakteristika: Zum einen ist keines seiner Argumente neu. Diese entstammen der seit 100 Jahren vorgetragenen, modernitätskritischkonservativen Medienkritik, in diesem Fall konzentriert auf die darin geführte, populäre Gewaltdebatte. Deren Anhänger finden sich auf diese Weise von Grossman/DeGaetano dort abgeholt, wo sie stehen. Zum zweiten trifft das Büchlein eine mediale Umbruchssituation. Bei Postman war das hierzulande in den 1980er Jahren die Verbreitung des kommerziellen Fernsehens mit seinen noch unvertrauten, vor allem von den Jüngeren genutzten Angeboten. Nun ist es die digitale Revolution und darin das Aufkommen von PC- und Videospielen, die erneut vor allem von den jungen Generationen genutzt werden und ebenfalls bei den älteren, gegenüber den Spielen nachhaltig abstinent bleibenden Generationen Besorgnis auslösen. Wie es in den 1980ern Postman tat, so scheinen jetzt Grossman/DeGaetano die Berechtigung dieser Besorgnis nachzuweisen - und damit Orientierung ohne Zumutung der eigenen Beschäftigung mit der Sache selbst und gegebenenfalls der Selbstkorrektur zu liefern. Zum dritten aber bieten Grossman/DeGaetano Kritikweisen an, die aus dem Ghetto der konservativen Modernitätskritik zu befreien scheinen: Ähnlich Postman argumentieren sie ökonomiekritisch gegen eine verderbliche, allein profitorientierte Medienindustrie und holen so auch eher linksliberale Besorgnisträgerlnnen ab. Dass dies zumindest oberflächlich mit militärkritischen Positionen verbunden erscheint, unterstützt diesen Effekt. Ihm verdankt Grossman deshalb schon 1999 einen Artikel in der "Zeit“ (Die Zeit Nr. 39, 30.09.1999, Beilage "Leben“), der inm bereits drei Jahre vor der deutschen Übersetzung seines Büchleins hierzulande Popularität unter Intellektuellen verschafft.

Diese hat seine Schrift jedoch nicht verdient. Die Kernthese ist denkbar einfach und undifferenziert: Gewalt und Kriminalität in der Realität werden kausal durch die Gewaltinszenierung in Film und Fernsehen sowie durch virtuelle Spiele bewirkt. Der Prozess erfolgt in zwei Stufen: Film und Fernsehen desensibilisieren gegenüber Gewalt und erzeugen „das Verlangen und/oder den konditionierten Reflex (...), sich ohne Reue gewalttätig zu verhalten" (Grossman/DeGaetano 2002, 61). Spiele erlauben dann aber nicht etwa, dieses Verlangen sozialverträglich auszuleben - was im Denkschema Grossman/DeGaetanos auch spekulativ behauptet werden könnte -, sondern bewirken die Fertigkeit, zielsicher zu töten (ebd. 61, 85-91). Im Verbund erzeugen Film, Fernsehen und PC-/Videospiele Mörder und Amokläufer (ebd.). Die Medien haben dabei die primäre Wirkungsposition (ebd. 17), treten also nicht etwa erst sekundär als Verstärkungsmodi zu personalen Interaktionsverhältnissen als Sozialisationsfaktoren hinzu.

Grossman/DeGaetano verfechten damit schlankweg die alte Stimulus-Response-These. Nutzungsweisen, -kontexte und -motive spielen keine Rolle, ebenso wenig die Subjektposition der Nutzenden gegenüber Medienprodukten. Das Buch ist im Vergleich zur Publikumsforschung - wie sie hierzulande etwa von Roland Eckert, Waldemar Vogelgesang oder Rainer Winter zum Film und von J ürgen Fritz und Wolfgang Fehr zu Spielen vorgelegt worden ist - von einer geradezu drastischen Unkenntnis. Gleichwohl sehen Grossman/DeGaetano auch keinen Anlass, sich für die Aneignungsformen der Publika und deren soziale Differenzierung zu interessieren. Die Nutzenden nämlich gelten innen als schrankenlos manipulierte, plastische Masse, in die sich einformen lässt, was die Produkte enthalten.

Entsprechend eklektisch und peripher wird die Literatur zur neueren Wirkungsforschung eingesetzt: Herangezogen werden George Gerbner, Leonard Eron und Lowell Huesman sowie Brandon Centerwall, Autoren also, die auch in der deutschen Gewaltdebatte Konjunktur haben, jedoch umstritten sind. Wie verschiedene neuere deutsche Veröffentlichungen, zitieren Grossman/DeGaetano zudem die Studie von Tannis Williams: The Impact of Television, New York: Academic Press 1986, sinnentstellend: Aus einer Untersuchung zur Einführung des Fernsehens in ein zuvor fernsehloses Gebiet wird abgeleitet, dass gewalthaltige Programme eine Steigerung von Gewalt und Kriminalität zur Folge hätten. Gerade die Gruppe, bei der diese Veränderung festgestellt worden sein soll, hatte aber im Unterschied zu zwei Kontrollgruppen kein wirklich gewalthaltiges Programm rezipiert.

Ähnlich wenig wie die Mediennutzenden als Subjekte, interessieren Grossman/DeGaetano die entsprechenden Medienprodukte und Werke. So fordern sie zwar zunächst, die Aussagestruktur und damit die (auch ethischen) Bedeutungszuschreibungen zu berücksichtigen, die Gewalt etwa in den Inszenierungen des Films je und je durch die werkimmanenten Kontexte erhält (ebd. 21). Sie unterstreichen sogar, dass dies - thesengemäß - entscheidend für die Art der Wirkung sei (ebd. 21). Erst gegen Ende des Buches aber kommen sie auf die Notwendigkeit 
solcher Differenzierung wieder zurück, indem sie diese erneut lediglich fordern (ebd. 109), ohne diese Forderung dazwischen selbst eingelöst zu haben. Auch nur der Ansatz zu einer Werkanalyse und zur Berücksichtigung von Aussagestrukturen spielt im gesamten Buch keine Rolle.

Ganz im Muster der populären Gewaltdebatte wird stattdessen lediglich auf das Vorhandensein von Gewalt abgestellt und die Häufigkeit von Gewaltakten ausgezählt (z.B. ebd. 51). Dabei kommen Grossman/DeGaetano zu Urteilen, wie dem, dass der Film "Dick Tracy“ gewaltverherrlichender und ethisch bedenklicher sei als „Death Wish“ („Ein Mann sieht rot"), weil im erstgenannten 14 Morde stattfänden, im zweitgenannten hingegen weniger (ebd. 47). Angesichts der Tatsache, dass „Death Wish“ über eine ausgefeilte emotionale Dramaturgie verfügt, die dem Publikum nun in der Tat nahe legt, individuelle Gewaltübung aus Rache und Selbstjustiz gut zu heißen, „Dick Tracy“ hingegen eine hochgradig verfremdende ComicVerfilmung ist, in der Gewalt einen realitätsfernenen, genretypischen Tabubruch darstellt, mutet dieses Urteil sachlich mehr als unangemessen an. Es entspricht freilich der Unbekümmertheit, mit der Grossman/DeGaetano Gewalt im Zeichentrick-, Problem-, Kriminal-, Action- und Horrorfilm als gleichbedeutend und -gefährlich behandeln. In der deutschen Übersetzung wird diese Undifferenziertheit nochmals gesteigert, da der von Grossman/DeGaetano benutze Terminus „violent" durchgängig mit "gewaltverherrlichend“ über tragen wird. Dass die Inszenierung von Gewalt kritisch sein kann, kommt nirgendwo in Blick; auch jene hochmoralischen Produkte, wie "The Untouchables" und "Gunsmoke“ ("Rauchende Colts"), in denen stets Law and Order und wertsicheres Verhalten empfohlen werden, gelten Grossman/DeGaetano lediglich als violent/gewaltverherrlichend (ebd. 40).

Im ganzen zeigt sich das Buch damit als ausgesprochen undifferenziertes, um nicht zu sagen: ideologisches Werk. Es sollte seinem Untertitel entsprechend bewertet und behandelt werden: als „Aufruf" - also als Pamphlet -, dem man wissenschaftlich und im institutionellen Handeln möglichst zurückhaltend und misstrauisch begegnen sollte. Vor allem wissenschaftlich sollte man aus der "Postmania" gelernt haben und eine "Grossmania“ vermeiden. 